\title{
EFEITOS DA AURICULOTERAPIA NA DOR E ANSIEDADE EM FUNCIONÁRIOS DE UMA UNIDADE BÁSICA DE SAÚDE: UM RELATO DE EXPERIÊNCIA
}

Auriculotherapy effects on pain and anxiety among Basic Health Unit's employees: an experience report http://dx.doi.org/10.21116/2019.6

FREITAS, Luciana A. B.

Prefeitura Municipal de Campinas.

\section{BARAGATTI, Daniella Y.}

UNIFAJ, Prefeitura Municipal de Campinas

RESUMO: O Brasil é o país com a maior taxa de pessoas com transtornos de ansiedade no mundo e o quinto em casos de depressão. Juntos, fatores fisiológicos individuais e socioculturais, fatores socioeconômicos, como a atual crise econômica e desemprego, fatores ambientais, como o estilo de vida, acarretam em forte impacto sobre a saúde mental da população. Tendo em vista essa estreita relação entre condições de trabalho e saúde mental, torna-se imperativo a elaboração de estratégias que se adequem a atual realidade e assegurem ao servidor condições para exercício de suas obrigações, um atendimento de qualidade ao usuário e a consequente diminuição dos gastos com afastamentos. O presente estudo relata a aplicação de um tratamento preventivo de auriculoterapia protocolar para a equipe multiprofissional de uma unidade de atenção primária em Campinas/SP. Os participantes receberam 10 sessões de auriculoterapia com sementes, a cada três dias, durante o período de cinco semanas. A proposta de auriculoterapia mostrou-se importante ferramenta de cuidado a saúde do trabalhador, valorizando a saúde mental e física dos servidores. É necessário maior sensibilização de gestores para elaborar estratégias que visem a prevenção e a promoção de saúde dos próprios cuidadores, visando cada vez mais acolher o seu sofrimento.

Palavras-chave: Auriculoterapia; Ansiedade; Atenção Básica

ABSTRACT: Brazil holds the highest rate of people suffering from anxiety disorders in the world and ranks fifth in number of depression cases. Together, the individual and sociocultural physiological factors have a strong impact on the population's mental health. Given this close relationship between working conditions and mental health, it is imperative to develop strategies that fit the current reality and allow the employees to adequately exercise their obligations, while also ensuring quality care to the users and lowering the costs of an increasing absenteeism among these public servers. The present study reports the application of a preventive treatment of protocol auriculotherapy for a Basic Health Unit's multidisciplinary team, located in Campinas/SP. Participants received 10 sessions of auriculotherapy with seeds every 3 days, for a 5 week period. The proposal to use auriculotherapy in Primary Care givers was proved to be an important tool to care for the health of workers, one that improves the mental and physical health of employees. Increasing awareness among managers is needed to develop strategies more suited to the prevention of mental 
disorders and the increase in the overall health of caregivers themselves, strategies that seek to embrace and treat their suffering.

Key Words: Auriculotherapy, Anxiety, Primary Health Care

\section{INTRODUÇÃO}

O Brasil é o país com a maior taxa de pessoas com transtornos de ansiedade e o quinto em casos de depressão. Segundo relatório lançado em fevereiro de 2017 pela Organização Mundial da Saúde (OMS), 9,3\% dos brasileiros têm algum transtorno de ansiedade e 5,8\% da população sofre de depressão (OMS, 2017). Ademais, dados da Secretaria de Previdência afirmam que os afastamentos por transtornos de ansiedade tem crescido significativamente, totalizando 26,5 mil casos em 2016 (ASSOCIAÇÃO NACIONAL DE MEDICINA DO TRABALHO, 2018).

Além de fatores fisiológicos individuais e socioculturais, fatores socioeconômicos, como a atual crise econômica e desemprego, e fatores ambientais, como o estilo de vida em grandes cidades, acarretam em forte impacto sobre a saúde mental da população. O cenário político e econômico do país tem contribuído para a expansão de uma sensação, cada vez mais generalizada, de ansiedade e insegurança (ROQUE, 2015).

Esses aspectos também impactam a qualidade de vida do servidor público, que embora disponha de alguma estabilidade profissional, tem de enfrentar a constante precarização do seu ambiente e estrutura de trabalho, fruto de políticas econômicas de austeridade cada vez mais intensas. O funcionalismo público enfrenta hoje problemas como a falta de estrutura material básica para desempenho de suas obrigações e uma constante falta de funcionários, que leva ao acúmulo de funções e produz nos servidores uma sensação de responsabilidade pelo sucateamento dos serviços ofertados a população (NUNES, 2009).

A morbimortalidade tendencial da população trabalhadora aponta para uma prevalência cada vez mais freqüente de agravos caracterizados por um mal estar difuso e por doenças que ocorrem na população geral, mas que entre os trabalhadores passam a ocorrer em faixa etária mais precoce (SELLIGMANNSILVA, 1997). As políticas públicas direcionadas à saúde do trabalhador tendem 
a ter como foco principal os trabalhadores vinculados às organizações privadas, deixando uma importante lacuna na atenção à saúde para os servidores públicos (REMMINGER, 2007) Entende-se os processos de trabalho a partir de uma perspectiva que os considere como atividade que não é neutra em relação à saúde, podendo favorecê-la ou não.

Estudos demonstram que profissionais de saúde são mais suscetíveis a desenvolver doenças físicas e mentais no trabalho, em larga medida devido a elevada carga de trabalho associada à assistência de pacientes em constante dor e sofrimento. Essa predisposição associada a piora das condições de trabalho mencionadas anteriormente, pode gerar dores e ansiedade no trabalhador, o que tem elevado os índices de absenteísmo (CINTRA et al, 2009).

Dor pode ser definida como uma experiência emocional desagradável, associada a uma real ou virtual lesão tecidual. Possui característica subjetiva e multidimensional, podendo envolver aspectos físicos e emocionais. Sempre gera um impacto negativo na qualidade de vida do indivíduo, inclusive afetando o seu desempenho no trabalho (CINTRA et al, 2009). Já ansiedade pode ser considerada como "vago e incômodo sentimento de desconforto ou temor, acompanhado por resposta autonômica (a fonte é frequentemente não específica ou desconhecida para o indivíduo); sentimento de apreensão causado pela antecipação de perigo" (NANDA INTERNATIONAL, 2014).

A auriculoterapia integra um conjunto maior de técnicas terapêuticas fundamentadas nos conceitos da Medicina Tradicional Chinesa (MTC). É uma prática oriental popular em diversos países e tem sido largamente utilizada na assistência à saúde, tanto nos aspectos preventivos quanto curativos (SOUZA, 2012). Várias teorias são descritas afim de explicar os benefícios da acupuntura, no entanto, seus mecanismos de ação ainda não são totalmente explicados pela medicina ocidental.

Pela teoria do sistema reflexo o estímulo dos pontos do pavilhão auricular, é capaz de tratar doenças físicas e mentais pois exerce impacto no Sistema Nervoso Central e, consequentemente, no organismo como um todo, devido ao reflexo gerado por diversos filetes nervosos e vasos capilares (MOURA et al, 2015).

Tendo em vista essa estreita relação entre condições de trabalho e saúde mental, torna-se imperativo a elaboração de estratégias que se adequem 
a atual realidade e assegurem ao servidor condições adequadas para exercício de suas obrigações, um atendimento de qualidade ao usuário e a consequente diminuição dos gastos com afastamentos.

A Unidade Básica de Saúde (UBS) caracteriza-se, conforme a Política Nacional de Atenção Básica (PNAB, 2011), como a principal porta de entrada do sistema de saúde. Sua função é realizar atendimentos de atenção básica e integral à um número definido de famílias localizadas em área geográfica delimitada, apresentando grau de descentralização e capilaridade. Além disso, tem o dever de promover a continuidade do cuidado, organizando o fluxo de encaminhamento para os demais níveis de atendimento quando se fizer necessário. A partir de todas essas ações, as equipes estabelecem vínculo com a população, possibilitando o compromisso e a co-responsabilidade dos profissionais com os usuários e a comunidade (VILELA, 2015 e BRASIL, 2011).

De acordo com o material de Formação em Auriculoterapia para profissionais de saúde da Atenção Básica (UNIVERSIDADE FEDERAL DE SANTA CATARINA, 2016), a prática de auriculoterapia pode ser eficaz no tratamento e prevenção de doenças entre esses profissionais, assim como na promoção de saúde e integração da equipe como um todo. Trata-se de uma técnica de baixo custo e fácil aplicação, prevista na Política Nacional de Práticas Integrativas e Complementares (PNIC). O Ministério da Saúde nomeou de Práticas Integrativas e Complementares as Medicinas Alternativas e Complementares, como são denominadas na literatura científica internacional (Complementary and Alternative Medicine). A PNPIC, além de legitimar as práticas da fitoterapia, da homeopatia, da medicina tradicional chinesa, medicina antroposófica e do termalismo social, impulsionou o reconhecimento pelo SUS de todas as demais PIC (BRASIL, 2006).

A auriculoterapia configura-se como um método de terapia passível de ser aplicado como tratamento prioritário ou ainda usado como complemento de um outro tratamento, melhorando sua eficiência (MOURA, 2015). Neste contexto, o trabalho teve como objetivo apresentar um relato de experiência de um tratamento preventivo de auriculoterapia com sementes, para a equipe multiprofissional de uma UBS. 


\section{METODOLOGIA}

Trata-se de um estudo descritivo, tipo relato de experiência. Conforme a tipologia de Gil, o estudo descritivo tem o objetivo de descrever determinado fenômeno e estabelecer associações entre suas variáveis (GIL,2008). Neste relato de experiência abordou-se aspectos de uma intervenção prática realizada em uma Unidade Básica de Saúde, apresentando um recorte sobre seus benefícios e desafios.

A pesquisa foi desenvolvida pela residente fisioterapia, que possui formação básica em auriculoterapia na Atenção Primária à Saúde, em uma unidade de Atenção Primária da região sudoeste de Campinas/SP.

Baseado no protocolo APPA (The Auricular Protocol for Pain and Anxiety) para dor e ansiedade, desenvolvido por pesquisadores Northen College of Acupunture, no Reino Unido (KUREBAYASHI et al, 2017), o presente estudo buscou relatar a experiência da implementação dessa prática interativa na equipe multiprofissional do Centro de Saúde Jardim Capivari, através de um diário de campo.

Ao longo de 5 semanas consecutivas foram realizadas 10 sessões de auriculoterapia com sementes de mostarda, 2 vezes por semana. Realizadas no local de trabalho, com duração de 5 a 10 minutos. Os pontos utilizados neste protocolo foram: Shenmen, tranquilizante, tálamo, sistema simpático e ponto zero, conforme Figura 1. A aplicação foi realizada unilateralmente em cada sessão.

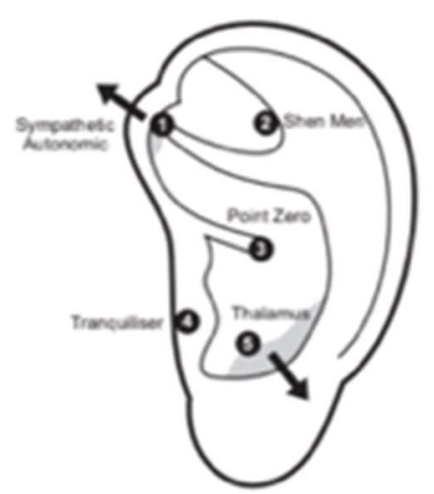

iscepiFigura 1: Protocolo Auricular para Dor e Ansiedade (APPA)

Fonte: http://www.appa-acupuncture.org/ 
Os riscos relacionados foram os envolvidos na aplicação de auriculoterapia com sementes, como: dor, vermelhidão local, prurido e pequenas escoriações. Uma lista ficou disponível para preenchimento dos funcionários que manifestassem interesse em participar da pesquisa. Inicialmente, foram listados 23 funcionários, incluindo residentes multiprofissionais, técnicos e auxiliares de enfermagem, técnicos de farmácia, dentistas, auxiliares de saúde bucal, agentes comunitários de saúde, técnicos administrativos e auxiliares de limpeza (18 mulheres e 5 homens, com idade média de 38 anos - 23 a 54 anos). No entanto, 2 funcionárias não puderam participar por estarem grávidas, 2 indivíduos não concluíram por apresentar prurido ao micropore utilizado e houve 1 abandono na $3^{a}$ semana de intervenção por motivo de licença médica. Beneficiaram-se do estudo, por tanto, 18 funcionários, conforme demonstrado pela figura 2.

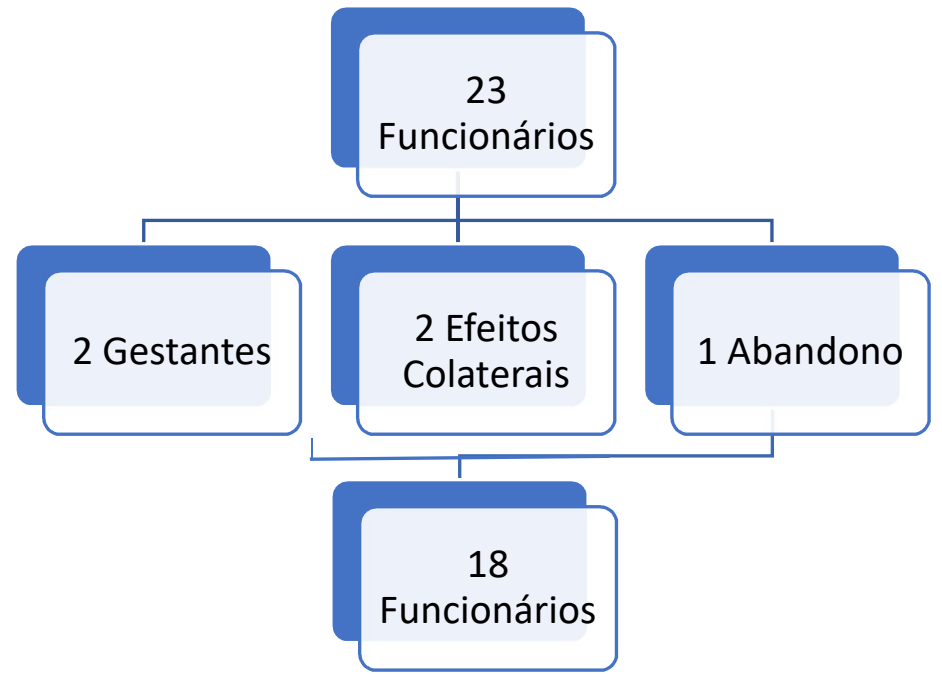

Figura 2. Amostra de indivíduos

Para o registro das informações observadas o pesquisador relatou as impressões em um Diário de Campo logo após as intervenções, objetivando minimizar a perda de informações relevantes. Em uma visão geral, os diários de campo ponderaram características particulares das práticas, como: data, local de realização, início e término da atividade, pontos mais dolorosos, relatos de sensações e sintomas, além de descrever as facilidades e desafios expressas em cada encontro. 
Realizou-se a localização dos pontos reativos com um apalpador manual, seguido de higienização do pavilhão auricular com álcool etílico 70\% e aplicação de sementes de mostarda com fita microporosa não alergênica nos referidos pontos.

A terapeuta foi uma fisioterapeuta residente, com formação básica em Auriculoterapia para profissionais de saúde da Atenção Básica, realizada pela Universidade Federal de Santa Catarina (Universidade Federal de Santa Catarina, Formação em Auriculoterapia para profissionais de saúde da Atenção Básica - MÓDULO I, 2016) e disponível aos profissionais da Atenção Primária pela Prefeitura Municipal de Campinas no ano de 2017.

Foram seguidas, para o desenvolvimento desse estudo, as diretrizes e as normas regulamentadoras de pesquisas que envolvem seres humanos, aprovadas pelo Conselho Nacional de Saúde - Resolução 466/12.

\section{RESULTADOS E DISCUSSÃO}

É comum que a demanda por ações voltadas a saúde e bem-estar dos funcionários sejam recorrentes e pertinentes, porém pouco se fala ou faz dentro do ambiente de trabalho. A oportunidade de receber um tratamento preventivo de auriculoterapia despertou interesse na maioria dos funcionários. Como a técnica também vem sendo empregada em tratamento de pacientes, alguns indivíduos já estavam familiarizados ou até mesmo já haviam realizado sessões de auriculoterapia, o que pode ter facilitado a adesão à pesquisa.

Os funcionários interessados e que se encaixaram nos critérios de inclusão, foram questionados sobre sintomas de ansiedade e níveis de dores em geral. Todos afirmaram apresentar algum tipo de dor, principalmente as de origem músculo esqueléticas, como lombalgia e artralgias. Relataram também cansaço extremo, dificuldade para dormir e concentrar, pensamentos negativos frequentes. A maioria dos indivíduos em questão, não praticam nenhuma atividade física regularmente.

Um estudo realizado em João Pessoa (PB-Brasil) apontou que 19,40\% dos profissionais de ESF apresentaram risco de adoecimento mentaL (PASCOAL, 2008). No interior do Goiás, outro trabalho destacou que $30 \%$ dos 
profissionais apresentaram algum grau de ansiedade, destes, 20\% apresentaram ansiedade leve, 2\% moderado e 8\% grave (MOURA et al, 2018).

No Brasil, o campo da saúde do trabalhador tem uma bela história de pessoas, instituições e movimentos organizados, que lutaram e lutam, desde o movimento pela reforma sanitária na década de 1970, por sua concretização e institucionalização. Ao mesmo tempo, possui uma história de invisibilidade e isolamento. As primeiras ações de saúde pública, voltadas para a Saúde do Trabalhador; organizaram-se a partir da década de 1970, com equipes e serviços especializados em estreita integração com o movimento sindical, porém, muitas vezes, funciona como espaços isolados na rede de saúde (GOMES, 2018).

Quanto ao protocolo APPA, os pontos mais dolorosos à palpação durante as sessões foram o Shenmen e o ponto zero. O ponto Shenmen, localizado na fossa triangular do pavilhão auricular, é comumente utilizado como ponto analgésico, sedativo e anti-inflamatório (GARCIA, 2006). Em outros estudos, o ponto zero já foi utilizado em pós-operatório, juntamente com o Shenmen, como regulador da atividade parassímpatica, obtendo resultados positivos em relação a frequência cardíaca (ARAl et al, 2013).

Os melhores resultados, baseados nos relatos dos participantes, foram melhora da qualidade do sono, aumento da sede, alívio de dores, diminuição da ansiedade, maior disposição, e até mesmo aumento de libido. Observou-se também uma tendência dos participantes sugerirem pontos além dos determinados pelo protocolo, a medida que relatavam uma queixa específica, como por exemplo, alívio de sintomas da menopausa, dor de dente, sinusite, etc. No final da intervenção os funcionários questionavam quando poderiam participar novamente e alguns daqueles que, por algum motivo não puderam participar, também demonstraram interesse por uma nova intervenção. Em geral, percebeu-se que o interesse dos funcionários em relação a Medicina Tradicional Chinesa e Práticas Integrativas cresceu quando comparado ao início da intervenção.

A prática de auriculoterapia tornou-se, neste período, uma importante ferramenta de cuidado com o trabalhador. Além dos efeitos fisiológicos, buscouse também a criação de um espaço onde o indivíduo pudesse ser ouvido e acolhido em suas dores e angústias. Apesar do tempo de aplicação ter sido 
curto (de 5 a 10 minutos), o funcionário, que está sempre prestando cuidado ao usuário, desta vez pode ser também cuidado.

Um estudo realizado com estudantes de enfermagem, sugere que os efeitos significativos da auriculoterapia com sementes nos níveis de ansiedade foram observados até a $8^{\mathrm{a}}$ sessão. Essa melhora obtida manteve-se por 15 dias, porém em declínio (PRADO, 2012).

O estudo de Paschoa, Zanei e Whitaker (2007) apontou que é necessário investimento voltado a melhorar a qualidade de vida dos profissionais que atuam na área da saúde, em específico da enfermagem, pois, assim a própria instituição teria benefícios e contaria com profissionais mais satisfeitos, melhorando assim a produtividade e qualidade da assistência prestada. Além disso, Carvalho, Araújo e Bernades (2016) apontaram que o trabalho em excesso contém grande carga emocional, pois, lida com enfermidade e morte de indivíduos. Desse modo, os profissionais podem ter uma reação de incapacidade, o que favorece sofrimento. Foram apontadas algumas medidas que os profissionais realizam como forma de aliviar a ansiedade como: ouvir música (34\%) e a prática de caminhada (26\%).

A auriculoterapia tem sido cada vez mais disseminada na Atenção Básica através da implementação das PICS e conforme relatado neste estudo, pode configurar-se como uma ferramenta de grande potencial preventivo, valorizando a saúde mental e física dos servidores. Percebe-se que sendo possível atender o trabalhador quer seja na fase de prevenção ou tratamento, no que diz respeito a transtorno mental e outros transtornos que podem acometer a sua saúde, será possível diminuir o preconceito frente o adoecimento entre esta população, proporcionando assim, a melhora da qualidade de vida, a manutenção da qualidade da saúde física e mental, bem como a melhora do desempenho profissional e êxito em sua vida pessoal e social.

Embora o protocolo seja um passo importante na realização da pesquisa científica, as fórmulas rígidas nem sempre parecem estar em consonância com a perspectiva holística das práticas orientais clássicas. Uma pesquisa realizada com profissionais de enfermagem de um Hospital Universitário demonstrou resultados superiores na redução dos níveis de estresse no grupo sem protocolo, que recebia a auriculoterapia de maneira individualizada (KUREBAYASHI, 2015). No entanto, o uso protocolar pode facilitar a disseminação da prática por 
outros profissionais capacitados e difundir o uso da técnica, beneficiando mais pacientes.

Quanto às limitações do estudo, não se pode assegurar se houve participação adequada dos sujeitos que foram tratados com sementes, pois as sementes precisam ser pressionadas para alcançar melhores resultados.

Faz-se necessário maior envolvimento e capacitação de mais profissionais nesta prática para a ampliação da oferta da auriculoterapia na $A B$. Sugere-se assim, que os órgãos do Estado invistam efetivamente em programas de prevenção e promoção à saúde do trabalhador, uma vez que a execução de campanhas relacionadas à promoção da saúde, ao bem-estar físico e mental, à qualidade de vida do trabalhador, à prevenção de lesões e doenças e à integração dos trabalhadores.

\section{CONSIDERAÇÕES FINAIS}

A prática de auriculoterapia tem sido cada vez mais disseminada na Atenção Básica através da implementação das PICS e conforme relatado neste estudo, pode configurar-se como uma ferramenta de grande potencial preventivo, valorizando a saúde mental e física dos servidores. Promover saúde nos locais de trabalho implica, necessariamente, fortalecimento da capacidade individual e coletiva para transformar as situações que agridem e fazem sofrer. É necessário priorizar nos locais de trabalho a afirmação da vida e a luta pela saúde.

Este trabalho buscou também agregar na sensibilização de gestores e demais profissionais responsáveis por elaborar estratégias que visem a prevenção e a promoção de saúde dos próprios cuidadores/funcionários, visando cada vez mais acolher o sofrimento deste trabalhador.

Sabe-se que não há maneiras para remover do ambiente de trabalho todos os fatores facilitadores ou desencadeadores de transtornos mentais e comportamentais. Porém, é importante que os órgãos do Estado invistam efetivamente em programas de saúde do trabalhador e qualidade de vida, pois isso pode proporcionar um ambiente menos desgastante a estes indivíduos e consequentemente, oferecer um melhor serviço aos usuários. 


\section{REFERÊNCIAS}

ARAI, Y.C.P.; SAKAKIMA, Y.; KAWANISHI, J.; NISHIHARA, M.; ITO, A.; YUSUKE, T. et al. Auricular Acupuncture at the "Shenmen" and "Point Zero" Points Induced Parasympathetic Activation. J Evid Based Complementary Altern Med, 2013

Associação Nacional de Medicina do Trabalho. "Cresce números de afastamentos por transtornos de ansiedade" Disponível em < https:// www.anamt.org.br/portal/2017/07/12/cresce-numero-de-afastamentos-por-transtornosde- ansiedade/ > Acessado em: 19 ago. 2018

Brasil. Ministério da Saúde. Política Nacional de Práticas Integrativas e Complementares (PNPIC). Brasília, 2006.

BRASIL, V.V.; ZATTA, L.T.; CORDEIRO, J.A.B.L. ET AL. Qualidade de vida de portadores de dores crônicas em tratamento com acupuntura. Rev. Eletrônica de Enfermagem. 2008; 10(2): 383-394.

BRASIL. Ministério da Saúde. Portaria n 2.488, de 21 de outubro 2011. Brasília, 2011.

CARVALHO, D.B.; ARAÚJO, T.M.; BERNARDES, K.O. Transtornos mentais comuns em trabalhadores da Atenção Básica à Saúde. Rev. Bras. Saúde Ocup. 2016

CINTRA, H.D.E.; SOUSA, A.A.E.; LAZZAROTTO, E.M.; MEZA, S.K.L.; KURMANN, R.A.S.; COSTA, E.S. et al. Fatores que prejudicam o trabalho do enfermeiro que atua em hospital. Seminário Internacional "Experiências de Agendas 21: os desafios do nosso tempo". 2009. Paraná, Brasil.

GARCIA, E.G. Auriculoterapia Escola Huang Li Chun. São Paulo: Roca; 2006.

GIL, A. C. Como elaborar projetos de pesquisa. São Paulo: Atlas, 2002.

GOMEZ, C.M.; VASCONCELLOS, L.C.F; MACHADO, J.M.H. Saúde do trabalhador: aspectos históricos, avanços e desafios no Sistema Único de Saúde Ciênc. saúde colet. 23 (6) Jun 2018

INTERNATIONAL NANDA. Diagnósticos de enfermagem da NANDA: definições e classificação 2012-2014. Porto Alegre: Artmed.

KUREBAYASHI, L.F.S.; SILVA, M.J.P.; Auriculoterapia Chinesa para melhoria de qualidade de vida de equipe de Enfermagem Rev Bras Enferm, 68(1):117-23, 2015

KUREBAYASHI, L.F.S.; FUMIKO, S. et al . Auriculotherapy to reduce anxiety and pain in nursing professionals: a randomized clinical trial. Rev. Latino-Am.

Enfermagem, Ribeirão Preto, v. 25, e2843, 2017.

MOURA, C.D.; CARVALHO, C.C.; SILVA, A.M.; IUNES, D.H.; CARVALHO, E.C.; CHAVES, ÉD. Auriculoterapia efeito sobre a ansiedade. Revista Cubana de Enfermería, 2015 
MOURA A.; LUNARDI, R.; VOLPATO R.; NASCIMENTO V.; BASSOS T.; LEMES A. Fatores associados à ansiedade entre profissionais da atenção básica. Rev. Portuguesa de Enfermagem de Saúde Mental, 2018

NUNES, A. V. L.; LINS, S. L. B. Servidores públicos federais: uma análise do prazer e sofrimento no trabalho. Psicologia: Organização e Trabalho, 2009; 9(1): 51 67

OMS. ORGANIZAÇÃO MUNDIAL DA SAÚDE. Depression and Other Common Mental Disorders: Global Health Estimates. Geneva, 2017. Disponível em $<$ https://www.who.int/mental health/management/depression/pr evalence global health estimates/en/> Acesso em: 13 set. 2019

PASCHOA, S., ZANEI, S.S.V; WHITAKER, I.Y. Qualidade de vida dos trabalhadores de enfermagem de unidades de terapia intensiva. Acta Paulista de Enfermagem, 20(3), 305-310, 2007

PASCOAL, F. S. Síndrome de burnout entre profissionais de saúde da Estratégia Saúde da Família: Risco de adoecimento mental (Dissertação de Mestrado). Universidade Federal da Paraíba, João Pessoa, 2008

PRADO, J.M.; KUREBAYASHI, L.F.S.; SILVA M.J.P.; Auriculotherapy Effectiveness in the Reduction of Anxiety in Nursing Students Rev Esc Enferm USP; 46(5):1200$120 ; 2012$

REMMINGER, T. ; NARDI, H. C. Saúde do trabalhador: um (não) olhar sobre o servidor público Rev. Serv. Público Brasília, 2007, 58 (2): 213-226

ROQUE, L. O trágico legado da "Nova Matriz Econômica" - um resumo cronológico (com dados atualizados) 2015 Disponível em <https:// www.mises.org.br/Article.aspx?id=2120> Acessado em: 21 ago. 2018

SELIGMANN-SILVA, E. Saúde mental e automação: a propósito de um estudo de caso no setor ferroviário. Cadernos de Saúde Pública, 1997; 13(2):95-110

SOUZA, M.P. Tratado de Auriculoterapia. Brasília: Novo Horizonte; 2012.

UNIVERSIDADE FEDERAL DE SANTA CATARINA, Formação em Auriculoterapia para profissionais de saúde da Atenção Básica - MÓDULO I, 2016.

VILELA, M.F.G.; Da moça da vigilância ao Núcleo de Saúde Coletiva na unidade básica de saúde: o que há de novo no modelo assistencial de Campinas? Campinas: Faculdade de Ciências Médicas, Universidade Estadual de Campinas; 2005. 


\section{SOBRE OS AUTORES:}

\section{LUCIANA APARECIDA BALIEIRO FREITAS}

Fisioterapeuta, Especialista em Atenção Primária.

Telefone: (19) 997261164

E-mail: lucianabfreittas@gmail.com

\section{DANIELLA YAMADA BARAGATTI}

Doutora em Ciências da Saúde, Enfermeira da Estratégia Saúde da Família de Campinas- SP, Docente do curso de graduação em Enfermagem do Centro Universitário de Jaguariúna.

Telefone: (19) 981336348

E-mail: danybaragatti@gmail.com 